\title{
Utilidad del índice de masa corporal en pacientes con enfermedad renal crónica
}

\author{
$M^{a}$ Eugenia Pons Raventos ${ }^{1}$, Ana Rebollo Rubio ${ }^{2}$, Rosario Amador Coloma $^{1}$ \\ ${ }^{1}$ Fresenius Medical Care Services Andalucía. Málaga. España \\ ${ }^{2}$ Unidad de Gestión Clínica de Nefrología. Hospital Regional Universitario de Málaga. Málaga. España
}

\section{Resumen}

Introducción: El índice de masa corporal es un indicador que se utiliza frecuentemente para identificar el sobrepeso y la obesidad en los adultos. En la población con enfermedad renal crónica, estudios de observación han reportado resultados contradictorios acerca de la asociación entre la obesidad y la mortalidad. También, diferentes autores, han observado como aquellos que presentan un índice de masa corporal elevado, demuestran un mejor estado nutricional.

Objetivo: Analizar el índice de una masa corporal de los pacientes en hemodiálisis y su relación con las complicaciones.

Material y Método: Estudio observacional, analítico y longitudinal. La muestra estuvo compuesta por 170 pacientes prevalentes en tratamiento con hemodiálisis o hemodiafiltración online.

Se calculó el índice de masa corporal en base al peso real y la talla del paciente en el momento de la recogida de datos. Se registraron los pacientes que causaron éxitus durante el periodo de seguimiento.

Resultados: Los pacientes fallecidos presentaban un índice de masa corporal menor, aunque las diferencias no resultaron estadísticamente significativas. Los pacientes que cumplieron el objetivo de índice de masa

Correspondencia:

$\mathrm{M}^{\mathrm{a}}$ Eugenia Pons Raventos

Fresenius Medical Care Services Andalucía

Clínica San Antonio

Avda. Miguel de Cervantes, local 7-8

29200 Antequera. Málaga

E-mail: meugeniapons@hotmail.com corporal, presentaron también mayores niveles de sobrehidratación, un menor índice de tejido graso, menores niveles de transferrina en sangre, un mayor $\mathrm{Kt} / \mathrm{v}_{\mathrm{e}} \mathrm{y}$ un mayor número de meses en terapia renal sustitutiva.

Conclusiones: Un índice de masa corporal $\geq 25 \mathrm{~kg} / \mathrm{m}^{2}$ no se asoció en nuestro caso con una mayor mortalidad, aunque si se observa una tendencia en los datos en ese sentido. Los pacientes con un índice de masa corporal $\geq 25 \mathrm{~kg} / \mathrm{m}^{2}$ presentaban un mejor estado nutricional.

PALABRAS CLAVE: índice de masa corporal; mortalidad; estado nutricional; índice de tejido graso; diálisis. Usefulness of body mass index in patients with
chronic kidney disease

\begin{abstract}
Introduction: The body mass index is an indicator that is often used to identify overweight and obesity in adults. In the population with chronic kidney disease, observational studies have reported contradictory results regarding the association between obesity and mortality. Also, different authors have observed as those having a body mass index higher, a better nutritional status is showed.
\end{abstract}

Objective: To establish a comparative study of subjects according to their body mass index.

Material and Method: Observational, analytical and longitudinal study. A total of 170 prevalent patients with chronic kidney disease on treatment with hemodialysis or online hemodiafiltration were recruited. The body 
mass index based on actual weight and size of the patient at the time of data collection was calculated. Patients who died during the follow-up period were recorded.

Results: The patients who died had a lower body mass index, although the differences were not statistically significant. Patients who met the target body mass index also had higher levels of hydration, a lower rate of fat, and lower blood levels of transferrin, a greater $\mathrm{Kt} / \mathrm{v}_{\mathrm{e}}$ and a greater number of months in renal replacement therapy.

Conclusions: A body mass index $\geq 25 \mathrm{~kg} / \mathrm{m}^{2}$ was not associated, in our case, with higher mortality, although a trend in this direction is observed. Patients with body mass index $\geq 25 \mathrm{~kg} / \mathrm{m}^{2}$ had a better nutritional status.

KEYWORDS: body mass index; mortality; nutritional status; fat tissue index; dialysis.

\section{Introducción}

La obesidad es una enfermedad altamente prevalente extendida en todo el mundo. La prevalencia de sobrepeso en España se estima que está en torno al 35,8\% y la de obesidad al $19,9 \%$. El índice de masa corporal (IMC) es un indicador simple de la relación entre el peso y la talla que se utiliza frecuentemente para identificar el sobrepeso y la obesidad en los adultos. La Organización Mundial de la Salud" (OMS) define que una persona tiene sobrepeso cuando posee un IMC igual 0 superior a 25 y obesidad cuando es igual o superior a 30. La obesidad es un factor de riesgo importante para las enfermedades cardiovasculares y renales, diabetes, algunos tipos de cáncer y para los trastornos musculoesqueléticos ${ }^{2-5}$. Además, se asocian con un mayor riesgo de mortalidad por cualquier causa ${ }^{6}$. Sin embargo, en la población con enfermedad renal crónica (ERC), estudios observacionales han reportado resultados contradictorios acerca de la asociación entre la obesidad y la mortalidad. Los estudios previos de personas en hemodiálisis (HD) han sugerido una "paradoja de la obesidad", donde ésta ejerce una acción protectora respecto a las diferentes causas de muerte, incluida la mortalidad por trastornos cardiovasculares ${ }^{7,8}$. Cuando hablamos de pacientes en HD, también se ha observado como aquellos que presentan un IMC elevado, demuestran un mejor estado nutricional, en comparación con pacientes con un IMC normal'.
El objetivo general del presente estudio ha sido analizar el índice de una masa corporal de los pacientes en hemodiálisis y su relación con las complicaciones.

\section{Material y Método}

Estudio observacional, analítico y longitudinal. Los criterios de inclusión fueron: pacientes adultos prevalentes con ERC en tratamiento de HD, que dieron su consentimiento para la participación en el estudio. Se excluyeron a aquellos pacientes con alguna patología aguda que precisara hospitalización en el mes previo a la recogida de datos. También se excluyeron a aquellos pacientes en los que estaba contraindicado el uso de bioimpedancia espectroscópica, para estimar la composición corporal, por ser portadores de un marcapasos unipolar, por episodio agudo de insuficiencia cardiaca descompensada o por criterio médico. La recogida de datos se inició durante el mes de octubre de 2013 reclutando una muestra total de 194 pacientes prevalentes de las clínicas Fresenius Medical Care de Málaga.

Se recogieron variables sociodemográficas, variables relacionadas con la patología renal, tratamiento y terapia renal sustitutiva (TRS). También se recogió el Índice de Comorbilidad de Charlson (ICC) modificado por Bheddu para pacientes con ERC ${ }^{10}$. Además, se recogieron parámetros analíticos de interés, bioquímicos y hematológicos, dosis de diálisis, estado de hidratación y estado nutricional, con el fin de caracterizar la muestra. Se calculó el IMC en base al peso real y la talla del paciente en el momento de la recogida de datos.

Los pacientes se clasificaron en tres grupos según su grado de movilidad: deambula por sí mismo, deambula con ayuda o en silla de ruedas. Posteriormente para hacer más operativa esta variable, consideramos pacientes dependientes a aquellos que precisaban silla de ruedas 0 ayuda para desplazarse y como independientes a aquellos sujetos que deambulaban sin ayuda.

Variables obtenidas a través de bioimpedancia: Estado líquido (Sobrehidratación $(\mathrm{OH})$ en litros (I); sobrehidratación en comparación a un control sano de composición corporal idéntica), volúmenes de fluidos, composición corporal, estado nutricional (índice de tejido magro (LTI) en $\mathrm{Kg} / \mathrm{m}^{2}$ e índice de tejido graso (FTI) en $\mathrm{Kg} /$ $\left.\mathrm{m}^{2}\right)$, y ángulo de fase a $50 \mathrm{kHz}$. En todos los pacientes se realizó la medición de la composición corporal utilizando el sistema Body Composition Monitor $\left(\mathrm{BCM}^{\circledR}\right)$, previo a la sesión de diálisis. Para la medición se retiraron todos los objetos metálicos que portaba el paciente, 
se evitó el contacto del personal con el sillón durante la medición, el paciente se colocó decúbito supino con las extremidades separadas al máximo y sin los pies apoyados en el reposapiés, los electrodos se colocaron dos a dos en la mano y pie contra lateral al acceso vascular. La calidad de la medición se determinó por $Q$ que debía ser mayor del $80 \%$ y del error que debía ser menor al $50 \%$, repitiéndose la medición cuando no se alcanzaban dichos indicadores de calidad. Otro indicador de calidad deficiente se hace evidente cuando la fase de cálculo de la medición supera los 5 minutos; en este caso también se repitió la medición. Las determinaciones analíticas se extrajeron previamente a la sesión de diálisis, en la sesión intermedia de la semana.

\section{Análisis estadístico}

Las variables categóricas han sido descritas con frecuencia y porcentaje. Para las variables numéricas utilizamos media \pm desviación típica, si siguen una distribución normal, si no es así, mediana y rango (mínimo - máximo) o rango intercuartil (percentil 25 - 75, IQR). Hemos estudiado la normalidad de los datos aplicando la prueba de Kolmogorov - Smirnov. En el análisis univariante, cuando ambas variables son categóricas hemos contrastado su grado de independencia con la prueba Chi-Cuadrado $\left(\chi^{2}\right)$. En el caso de que ambas variables sean numéricas, el estadístico usado para explorar la posible asociación lineal ha sido el coeficiente de correlación de Pearson o de Spearman, en función de la normalidad de los datos. Para comparar medias de variables numéricas en dos grupos hemos empleado el estadístico $\mathrm{T}$ Student para muestras independientes cuando los datos eran normales, en caso contrario hemos empleado su equivalente no paramétrico como es la prueba $U$ de Mann - Whitney. Las diferencias se han considerado estadísticamente significativas para $p<0,05$ con un intervalo de confianza (IC) del $95 \%$. Para el análisis de los datos se utilizará el paquete estadístico SPSS Statistics Versión 21.

\section{Resultados}

Se seleccionaron para el estudio 170 pacientes, quedando excluidos 24 (uno por fallecimiento, uno por trasplante, tres por ingreso hospitalario, dos por ausencia vacacional en otros centros durante el periodo de estudio y 17 por contraindicación médica a la medición de bioimpedancia). Del total de la muestra, el 60,6\% $(n=103)$ eran hombres y el $39,4 \%(n=67)$ mujeres. Los datos obtenidos en la variable edad, se observa gran dispersión, se obtuvo con una mediana de 70 años $(60$ 79, IQR), y un rango entre 25 y 89 años. El periodo de seguimiento fue de mediana 426 días. Las causas más frecuentes de ERC fueron respectivamente la diabetes $(n=41,24,1 \%)$ y la etiología vascular $(n=41,24,1 \%)$. En relación con el número de meses en TRS, la media fue de 57,38 meses $\pm 37,35$. Solo el $11,8 \%(n=20)$ de los pacientes habían recibido un trasplante renal previo. La modalidad de TRS más frecuente para esta muestra fue la HD convencional $(n=118,69,4 \%)$ y el acceso vascular prevalente la fístula arteriovenosa $(n=122$, $71,8 \%$ ). Los datos obtenidos con respecto al ICC se encuentran algo dispersos, siendo la mediana 4 (3 6, IQR). Según la división propuesta por Beddhü ${ }^{10}$, en nuestro grupo de estudio, el 25,3\% ( $n=51)$ presentaban una comorbilidad alta (ICC $=6-7$ ) o muy alta (ICC > 7). En cuanto al grado de movilidad, el $73,5 \% \quad(n=125)$ de los sujetos podían deambular por sí mismos, frente al $13,5 \%(n=23)$ que precisaba ayuda o el $12,9 \% \quad(n=22)$ que utilizaba silla de ruedas.

El $26,5 \%(n=45)$ de los pacientes presentó un IMC óptimo, entre $20-25 \mathrm{~kg} / \mathrm{m}^{2}$, el $41,65 \%(n=71)$ se encuentra entre $25-30 \mathrm{~kg} / \mathrm{m}^{2}$, el $27,65 \%(n=47)$ presentaron un IMC igual o superior a $30 \mathrm{~kg} / \mathrm{m}^{2}$, el porcentaje de pacientes que obtuvo un IMC entre 18,5 y 20 fue del $2,4 \%(n=4)$ y en tres casos $(1,8 \%)$ encontramos un IMC por debajo de 18,5. Los pacientes que presentaron un IMC superior o igual a $25 \mathrm{Kg} / \mathrm{m}^{2}$ (Tabla 1), presentaron también menores niveles de $\mathrm{OH}$, un mayor FTI, mayores niveles de transferrina en sangre, un menor $\mathrm{Kt} / \mathrm{v}_{\mathrm{e}} \mathrm{y}$ un menor número de meses en TRS, todo ello de forma significativa, con respecto a los que cumplen el objetivo. Los datos muestran una tendencia donde los pacientes con etiología diabética presentan en un $92,7 \%$ un IMC por encima de la normalidad $(p=0,000)$.

Tras constatar una correlación existente entre el IMC y el FTI $(p=0,001)$ cuando analizamos toda la muestra, hemos observado que al dividir a la población de estudio en dos grupos basándonos en los criterios de sobrepeso marcados por la OMS, esta correlación lineal directa solo se mantiene en el grupo de pacientes con un IMC $\geq$ $25 \mathrm{~kg} / \mathrm{m}^{2}(p=0,001)$ (Figura 1), dejando de ser significativa para el grupo de pacientes con un IMC $<25 \mathrm{Kg} /$ $m^{2}(p=0,154)$ (Figura 2).

Tras un máximo de 14 meses de seguimiento, tras comparar los datos basales, observamos como los pacientes fallecidos presentaban un IMC menor $(26,66 \pm 6,27)$ frente a los pacientes que permanecían vivos $(27,82 \pm$ $5,33)$, aunque las diferencias no resultaron estadísticamente significativas $(p=0,337)$. 
Tabla 1. Diferencias observadas entre los pacientes con relación al índice de Masa Corporal.

\begin{tabular}{|c|c|c|c|}
\hline & $\begin{array}{c}\text { IMC }<25 \mathrm{Kg} / \mathrm{m}^{2} \\
\mathrm{n}=52\end{array}$ & $\begin{aligned} \text { IMC } & \geq 25 \mathrm{Kg} / \mathrm{m}^{2} \\
\mathrm{n} & =118\end{aligned}$ & Significación \\
\hline Edad $\dagger$ & $68(25-84)$ & $70,5(25-89)$ & $p=0,259$ \\
\hline Sexo (hombre) & $37(35,9 \%)$ & $66(64,1 \%)$ & \multirow{2}{*}{$p=0,061$} \\
\hline Sexo (mujer) & $15(22,4 \%)$ & $52(77,6 \%)$ & \\
\hline Movilidad (independiente) & $34(27,2 \%)$ & $91(72,8 \%)$ & \multirow{2}{*}{$p=0,110$} \\
\hline Movilidad (dependiente) & $18(40 \%)$ & $27(60 \%)$ & \\
\hline Etiología diabética & $3(7,3 \%)$ & $38(92,7 \%)$ & $p=0,001$ \\
\hline Etiología vascular & $12(29,3 \%)$ & $29(70,7 \%)$ & $p=0,833$ \\
\hline Número de meses en TRS & $69,9 \pm 37,6$ & $51,9 \pm 36$ & $p=0,003$ \\
\hline Trasplante previo (sí) & $10(50 \%)$ & $10(50 \%)$ & \multirow{2}{*}{$p=0,068$} \\
\hline Trasplante previo (no) & $42(28 \%)$ & $108(72 \%)$ & \\
\hline FAV o prótesis vascular & $39(31,2 \%)$ & $86(68,8 \%)$ & \multirow{2}{*}{$p=0,773$} \\
\hline CVC & $13(28,9 \%)$ & $32(71,1 \%)$ & \\
\hline Modalidad TRS (HDF online) & $10(19,2 \%)$ & $42(80,8 \%)$ & \multirow{2}{*}{$p=0,033$} \\
\hline Modalidad TRS (HD) & $42(35,6 \%)$ & $76(64,4 \%)$ & \\
\hline Índice de Charlson † & $4(2-9)$ & $5(2-9)$ & $p=0,087$ \\
\hline Índice masa corporal $\left(\mathrm{Kg} / \mathrm{m}^{2}\right)$ & $22,05 \pm 1,87$ & $30,12 \pm 4,64$ & $p=0,001$ \\
\hline Sobrehidratación (I) & $1,49 \pm 1,29$ & $1,02 \pm 1,23$ & $p=0,026$ \\
\hline Índice de tejido magro $\left(\mathrm{Kg} / \mathrm{m}^{2}\right)$ & $10,41 \pm 2,03$ & $10,62 \pm 2,63$ & $p=0,577$ \\
\hline Índice de tejido graso $\left(\mathrm{Kg} / \mathrm{m}^{2}\right)$ & $12,3 \pm 4,29$ & $18,36 \pm 5,84$ & $p=0,001$ \\
\hline Ángulo de fase a $50 \mathrm{KHz}$ & $4,04 \pm 0,98$ & $4,2 \pm 0,96$ & $p=0,322$ \\
\hline Hemoglobina ( $\mathrm{g} / \mathrm{dl})$ & $11,30 \pm 1,09$ & $11,33 \pm 1,04$ & $p=0,868$ \\
\hline Transferrina (mg/dl) & $147,6 \pm 30,82$ & $163,5 \pm 33,71$ & $p=0,004$ \\
\hline Potasio $(\mathrm{mEq} / \mathrm{l})$ & $4,86 \pm 0,66$ & $4,84 \pm 0,63$ & $p=0,844$ \\
\hline Bicarbonato venoso $(\mathrm{mEq} / \mathrm{l})$ & $25,66 \pm 2,27$ & $25,27 \pm 2,11$ & $p=0,277$ \\
\hline Fósforo (mg/dl) & $4,15 \pm 1,37$ & $4,32 \pm 1,14$ & $p=0,404$ \\
\hline Colesterol (mg/dl) & $154,15 \pm 35,86$ & $151,68 \pm 33,92$ & $p=0,404$ \\
\hline Albúmina sérica $(\mathrm{g} / \mathrm{dl}) \dagger$ & $4(3-4,5)$ & $4,1(3-4,8)$ & $p=0,065$ \\
\hline $\operatorname{PCR}(\mathrm{mg} / \mathrm{L}) \dagger$ & $7,96(0,21-128,22)$ & $6,19(0,09-70,84)$ & $p=0,224$ \\
\hline Proteínas totales (g/dl) & $6,89 \pm 0,49$ & $6,93 \pm 0,57$ & $p=0,670$ \\
\hline Npcr & $1,07 \pm 0,15$ & $1,01 \pm 0,16$ & $p=0,068$ \\
\hline $\mathrm{eKt} / \mathrm{v}$ & $1,69 \pm 0,34$ & $1,55 \pm 0,28$ & $p=0,007$ \\
\hline
\end{tabular}

Las variables numéricas vienen expresadas como media \pm desviación típica y el estadístico utilizado es la T de Student cuando los datos siguen una distribución normal, en caso contrario hemos empleado su equivalente no paramétrico $U$ de Mann - Whitney $\dagger$. Las variables numéricas que no siguen una distribución normal se han descrito con mediana y rango intercuartil (percentil 25 - 75). Las variables categóricas se han expresado con frecuencia y porcentaje y han sido analizadas con el estadístico Chi-Cuadrado $\left(\chi^{2}\right)$. Índice de Masa Corporal (IMC). Tratamiento renal sustitutivo (TRS). Fístula arteriovenosa (FAV). Cáteter Venoso Central (CVC). Hemodiafiltración online (HDF online). Hemodiálisis (HD). Proteína $C$ reactiva (PCR). Tasa de catabolismo proteico (nPCR). Dosis de diálisis (Kt/ve $)$.

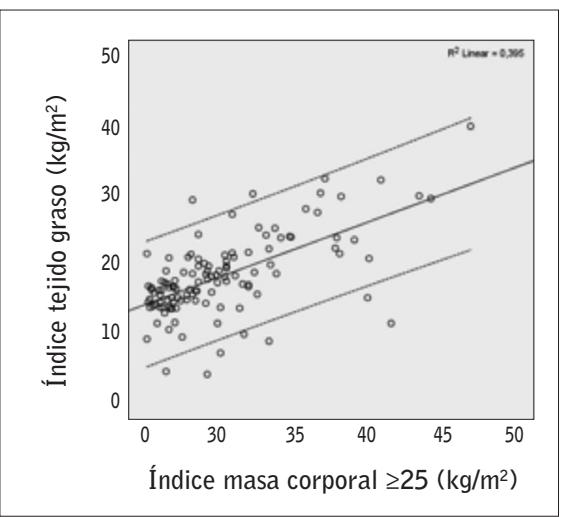

Figura 1. Regresión lineal entre el Índice de masa corporal y el índice de tejido graso medido por bioimpedancia. La línea continua representa la recta de regresión y las discontinuas representan los intervalos de confianza del 95\%. $\mathrm{R}^{2}=0,395$; $\mathrm{p}=0,001$.

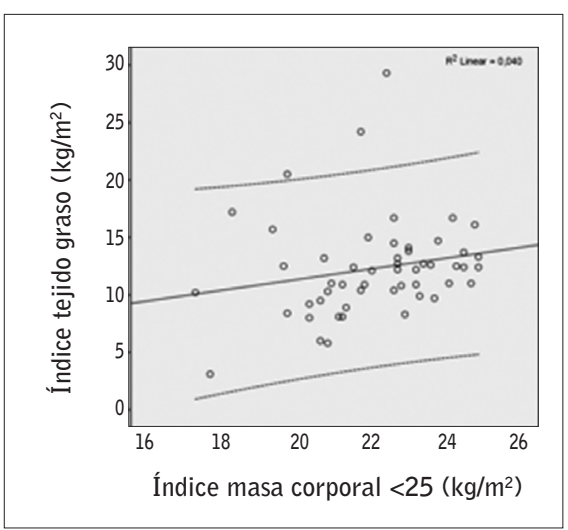

Figura 2. Regresión lineal entre el Índice de masa corporal y el índice de tejido graso medido por bioimpedancia. La línea continua representa la recta de regresión y las discontinuas representan los intervalos de confianza del 95\%. $\mathrm{R}^{2}=0,040$; $p=0,154$. 


\section{Discusión}

El concepto de promedio social fue concebido por Lambert Adolphe Jacques Quetelet (1796-1874) astrónomo y estadístico. Su objetivo inicial fue determinar las características de un "sujeto medio" y la distribución poblacional de las diferentes características humanas alrededor de dicho sujeto. En 1972, Keys et $\mathrm{al}^{11}$ popularizó el Índice de Quetelet en los estudios basados en la población. Se refirieron a él como "índice de masa corporal".

Alrededor de 1995, la OMS publicó la clasificación de peso corporal sobre la base del IMC y que posteriormente fue adoptado ampliamente ${ }^{12}$. EI IMC es un parámetro antropométrico de utilidad en pacientes con $\mathrm{ERC}^{13}$, ya puede proporcionar información valiosa, especialmente si se realizan mediciones seriadas. En múltiples estudios ha sido descrito ampliamente la relación entre un IMC alto y un mayor riesgo de muerte, pero ¿Qué sucede en los pacientes con ERC? En una revisión sistemática publicada en 2016, los autores afirman que sus hallazgos sugieren que la obesidad tiene un efecto protector para todas las causas de muerte en los pacientes en prediálisis y HD, pero no en los receptores de trasplantes. Sin embargo, afirman su relación es poco probable que sea lineal, con un probable mayor riesgo de muerte en las categorías extremas del $\mathrm{IMC}^{14}$. Observaron cómo los pacientes en diálisis con un IMC $>25 \mathrm{~kg} / \mathrm{m}^{2}$, experimentaron una reducción del $25 \%$ en todas las causas de mortalidad, en comparación con aquellos con un IMC < $25 \mathrm{~kg} / \mathrm{m}^{2,14}$ La mortalidad, en nuestro grupo de estudio, no se asoció al IMC, aunque observamos como los pacientes fallecidos presentaron un IMC menor, no siendo las diferencias estadísticamente significativas.

EI FTI medido por bioimpedancia, que representa el contenido de grasa del cuerpo, correlacionó de forma positiva y significativa con el IMC cuando analizamos toda la muestra $(r=0,683 p=0,001)$, al igual que otros autores ${ }^{15}$. Pero esta correlación no se mantuvo cuando dividimos la muestra y analizamos solo a los pacientes con un IMC $<25 \mathrm{Kg} / \mathrm{m}^{2}$. Un problema particular con el IMC como un índice de la obesidad es que no diferencia entre la masa corporal magra y la masa grasa $^{16-18}$. La relativamente pobre correlación entre el porcentaje de masa grasa corporal y el IMC también ha sido demostrado, en la base de datos National Health and Nutrition Examination Survey (NHANES III) en la que se utilizó la bioimpedancia para estimar el componente de grasa de la composición corporal. En sujetos con un IMC de $25 \mathrm{~kg} / \mathrm{m}^{2}$, el porcentaje de grasa corporal en los hombres varió entre el $14 \%$ y el $35 \%$, y en las mujeres entre el $26 \%$ y el $43 \%{ }^{19}$.

Diversos estudios han constatado como pacientes en HD con IMC elevado, demuestran un mejor estado nutricional y una mayor supervivencia ${ }^{20-23}$. Beberashvili y colaboradores observaron como la albúmina y transferrina fueron significativamente más elevados en los grupos de IMC más altos, después de ajustar por edad, sexo y estado de la diabetes. El grupo con un IMC más alto tenía una mayor masa magra corporal $(p=0,001)$ y masa grasa $(p=0,0001)$ y un mayor ángulo de fase $e^{9}$. En nuestros resultados observamos también unos mayores niveles de albúmina, transferrina, ángulo de fase a $50 \mathrm{KHz}$, masa grasa y masa magra en los pacientes con mayor IMC, aunque las diferencias solo fueron significativas en el caso de la transferrima $(p=0,004)$ y la masa grasa $(p=0,001)$. Aunque muchos autores valoran el estado nutricional mediante el IMC, nuestro estudio demuestra que es necesario diferenciar entre aquellos casos que presentan un buen índice de masa libre de grasa de aqueIlos casos, incluso con un IMC alto, donde el índice de masa libre de grasa es escaso. Según Kalantar-Zadeh, los primeros se asocian a un ángulo de fase a $50 \mathrm{~Hz}$ elevado y presentan una supervivencia significativamente mejor que los segundos. Estos datos, descritos recientemente, establecen la necesidad de diferenciar los diferentes componentes de la composición corporal para valorar su efecto pronóstico ${ }^{24}$.

\section{Conclusiones}

Teniendo en cuenta las limitaciones del presente artículo al tratarse de un estudio observacional, podemos concluir diciendo que un IMC $\geq 25 \mathrm{~kg} / \mathrm{m}^{2}$ no se asoció en nuestro caso con una mayor mortalidad, aunque si se observa una tendencia en los datos en ese sentido. Los pacientes con un IMC $\geq 25 \mathrm{~kg} / \mathrm{m}^{2}$ presentaban un mejor estado nutricional.

EI IMC no diferencia entre la masa corporal magra y la masa grasa, por ello debe ser tomado con precaución si queremos obtener un indicador fiable de obesidad.

\section{El autor declara que no hay conflicto de interés.}


Recibido: 15 agosto 2017

Revisado: 30 agosto 2017

Modificado: 25 septiembre 2017

Aceptado: 1 octubre 2017

\section{Bibliografía}

1. López Sobaler AM, Aparicio A, Aranceta Bartrina J, Gil Á, González Gross M, Serra-Majem L, et al. Overweight and General and Abdominal Obesity in a Representative Sample of Spanish Adults: Findings from the ANIBES Study. Biomed Res Int. 2016; 2016:8341487.

2. Singh GM, Danaei G, Farzadfar $F$, Stevens GA, Woodward M, Wormser D, et al. The Age-Specific Quantitative Effects of Metabolic Risk Factors on Cardiovascular Diseases and Diabetes: A Pooled Analysis. PLoS One. 2013; 8(7):e65174.

3. Berrington de Gonzalez $A$, Hartge $P$, Cerhan JR, Flint AJ, Hannan L, MacInnis RJ, Moore SC, et al. Body-Mass Index and Mortality among 1.46 Million White Adults. N Engl J Med. 2010; 363(23):2211-9.

4. Wormser D, Kaptoge S, Di Angelantonio E, Wood AM, Pennells $L$, Thompson A, et al. Separate and combined associations of body-mass index and abdominal adiposity with cardiovascular disease: collaborative analysis of 58 prospective studies. Lancet. 2011; 377(9771):1085-95.

5. Lu Y, Hajifathalian K, Ezzati M, Woodward M, Rimm EB, Danaei G. Metabolic mediators of the effects of body-mass index, overweight, and obesity on coronary heart disease and stroke: a pooled analysis of 97 prospective cohorts with 1.8 million participants. Lancet. 2014; 383(9921):970-83.

6. Aune D, Sen A, Prasad M, Norat T, Janszky I, Tonstad S, et al. BMI and all cause mortality: systematic review and non-linear dose-response meta-analysis of 230 cohort studies with 3.74 million deaths among 30.3 million participants. BMJ. 2016; 4(353);i2156.

7. Fleischmann E, Teal N, Dudley J, May W, Bower $J D$, Salahudeen AK. Influence of excess weight on mortality and hospital stay in 1346 hemodialysis patients. Kidney Int. 1999; 55(4):1560-7.
8. Port FK, Ashby VB, Dhingra RK, Roys EC, Wolfe RA. Dialysis dose and body mass index are strongly associated with survival in hemodialysis patients. J Am Soc Nephrol. 2002; 13(4):1061-6.

9. Beberashvili I, Sinuani I, Azar A, Yasur H, Feldman $L$, Efrati $S$, et al. Nutritional and inflammatory status of hemodialysis patients in relation to their body mass index. J Ren Nutr. 2009; 19(3):238-47.

10. Beddhu S, Bruns FJ, Saul M, Seddon P, Zeidel ML. A simple comorbidity scale predicts clinical outcomes and costs in dialysis patients. Am J Med. 2000; 108(8):609-13.

11. Keys A, Fidanza F, Karvonen MJ, Kimura N, Taylor $\mathrm{HL}$. Indices of relative weight and obesity. J Chronic Dis. 1972; 25(6):329-43.

12. Physical status: the use and interpretation of anthropometry. Report of a WHO Expert Committee. World Health Organ Tech Rep Ser. 1995; 854:1-452.

13. Ruperto López M, Barril Cuadrado G, Lorenzo Sellares V. Guía de nutrición en Enfermedad Renal Crónica Avanzada (ERCA). Nefrol Publ Of Soc Esp Nefrol. 2008;28 Suppl 3:79-86.

14. Ladhani M, Craig JC, Irving M, Clayton PA, Wong G. Obesity and the risk of cardiovascular and all-cause mortality in chronic kidney disease: a systematic review and meta-analysis. Nephrol Dial Transplant. 2016 May 4;gfw075.

15. Yuste C, Abad S, Vega A, Barraca D, Bucalo L, Pérezde José $A$, et al. Valoración del estado nutricional en pacientes en hemodiálisis. Nefrologia. 2013; 33(2):243-9.

16. Wellens RI, Roche AF, Khamis HJ, Jackson AS, Pollock ML, Siervogel RM. Relationships between the Body Mass Index and body composition. Obes Res. 1996; 4(1):35-44.

17. Strain GW, Zumoff B. The relationship of weightheight indices of obesity to body fat content. J Am Coll Nutr. 1992; 11(6):715-8.

18. Flegal KM, Shepherd JA, Looker AC, Graubard $\mathrm{BI}$, Borrud LG, Ogden CL, et al. Comparisons of percentage body fat, body mass index, waist circumference, and waist-stature ratio in adults. Am J Clin Nutr. 2009; 89(2):500-8.

19. Romero Corral A, Somers VK, Sierra Johnson J, Thomas RJ, Collazo Clavell ML, Korinek J, et al. 
Accuracy of body mass index in diagnosing obesity in the adult general population. Int $\mathrm{J}$ Obes (Lond). 2008; 32(6):959-66.

20. Mohebi R, Simforoosh A, Tohidi M, Azizi F, Hadaegh F. Obesity Paradox and Risk of Mortality Events in Chronic Kidney Disease Patients: A Decade of Follow-up in Tehran Lipid and Glucose Study. J Ren Nutr. 2015; 25(4):345-50.

21. Park J, Ahmadi S-F, Streja E, Molnar MZ, Flegal KM, Gillen $D$, et al. Obesity paradox in end-stage kidney disease patients. Prog Cardiovasc Dis. 2014; 56(4):415-25.

22. Wang J, Zhou Y, Yuan W. Relationship between body mass index and all-cause mortality in hemodialysis patients: a meta-analysis. Zhonghua Nei Ke Za Zhi. 2012; 51(9):702-7.
23. Jialin W, Yi Z, Weijie Y. Relationship between body mass index and mortality in hemodialysis patients: a meta-analysis. Nephron Clin Pract. 2012; 121(34):c102-111.

24. Kalantar-Zadeh K, Kopple JD, Humphreys MH, Block G. Comparing outcome predictability of markers of malnutrition-inflammation complex syndrome in haemodialysis patients. Nephrol Dial Transplant. 2004; 19(6):1507-19.

Este artículo se distribuye bajo una Licencia Creative Commons Atribución-NoComercial 4.0 Internacional. https://creativecommons.org/licenses/by-nc/4.0/

\section{Open Access (c) () (9)}

